\title{
On-Line Model to Control Bead Height for Automatic GMA Welding Process
}

\author{
Joon Sik Son ${ }^{1}$, Jong-Pyo Lee², Min-Ho Park², Byeong-Ju Jin², and Ill-Soo Kim²* \\ ${ }^{1}$ Research Institute of Medium\& Small Shipbuilding, 1703-8 Yongang-ri, Samho-eup, \\ Yeongam, Jeonnam, 526-897, South Korea. \\ ${ }^{2}$ Department of Mechanical Engineering, Mokpo National University, 1666 Youngsan-ro, \\ Chungkye-myun, Muan-gun, Jeonnam, 534-729, South Korea. \\ *Corresponding author Tel: +82-61-450-2416, Fax: +82-61-452-6376 \\ ilsookim@mokpo.ac.kr
}

\begin{abstract}
Recently, not only automatic welders have replaced human welders in many welding applications, but also reasonable seam tracking systems are commercially available. However, fully adequate process control systems have not been developed due to a lack of reliable sensors and mathematical models that correlate welding parameters to the bead geometry for the automated welding process. In this paper, two on-line empirical models using multiple regression analysis are proposed in order to be applicable for the prediction of bead height as welding quality for automatic GMA (Gas Metal Arc) welding process. For development of the proposed models, an attempt has been made to apply for a several methods. For the more accurate prediction, the predicted variables are first used to the surface temperatures measured using infrared thermometers with the welding parameters (welding current, arc voltage) because the surface temperature are strongly related to the formation of the bead geometry. And the developed models are applied for prediction of bead height as welding quality.
\end{abstract}

Keywords: On-line empirical model, Optimization, Bead height, Automatic GMA welding, Welding quality

\section{Introduction}

Many analytical approaches to process model have been developed from heat transfer relationships, but these are regarded by Cook et al. [1] as being only reasonably accurate. Most practical models are developed statically or experimentally, and attempted to decouple the welding parameters. However, decoupling of welding parameters is extremely difficult since each parameter has at least some effect on the others. For instance, bead penetration may be changed with a variance in welding current level, but this may also induce unwanted changes in bead height or weld spatter. Other closed loop system has been developed by Smartt et al. [2] for real-time control of reinforcement area and cooling rate. Doumanidis et al. [3] have attempted to derive simple dynamic models in their attempt to control bead width, bead penetration, reinforcement area, heat affected zone and rate of cooling at the center line of the weld. Mathematical-based control systems are not suited to truly real-time adaptive control because of their inability to isolate the welding parameters, thereby resulting in excessive processing times. These systems are not capable of taking account of heuristics and the relative effect of each welding parameter. Mathematical systems are also inflexible to changes in hardware due to their rigid control laws, and consequently are unable to learn from successes and errors. However, it can only consider fuzzy logic or expert systems that are capable of a learning function. Thus, a real-time bead penetration monitoring system is also achieved. Recently an on-line tracking optimization scheme for sensor guided robotic manipulators by associating sensor information, manipulator dynamics and a path generator model has been proposed [4]. Feedback linearization-decoupling permits the use of linear Single-Input SingleOutput (SISO) prediction models for the dynamics of each robot joint. Scene interpretation of CCD-camera images generates spline fitted segments of future trajectory. In the sensor vision field, the proposed optimization criteria minimize errors between state variables from the prediction model and the state variables of the spline trajectory generator [5]. Experimental results on implementation of a CCD-camera guided hydraulic robot and a welding robot, demonstrated the practical relevance of the proposed approach.

This paper presents an on-line mathematical model for predicting bead height and for investigating the effects of various welding parameters using the empirical models (on-line interaction and on-line quadratic models). Based on the 
experimental results, the on-line multiple regression models for predicting bead height are established. Finally, an additional experiment is conducted to verify the optimal welding parameters obtained from the developed models.

\section{Experimental Procedures}

In this study, the bead height, an important role in determining the optimal welding conditions, is employed to study the welding quality. Therefore in this study, Contact Tip to Work Distance (CTWD), gas flow rate, welding speed, arc current, welding voltage were chosen to investigate the effects of welding parameters and develop the empirical models for predicting bead height as output parameter. Statistically designed experiments that are based upon factorial techniques, reduce costs and provide the required information about the main and interaction effects on the response factors. All other parameters except these were fixed.

The experimental data that included five process parameters on bead height at 2 levels were obtained by using a welding robot. The design matrix that has 32 experimental welding process has been run where each row corresponds to one experimental run with two replications. In this study, the mean of these replications was considered output parameters to utilize the development of empirical models. The $150 \times 200 \times 4.5 \mathrm{~mm}^{3} \mathrm{SS} 400$ materials and steel wire with a diameter of $1.2 \mathrm{~mm}$ were employed for the experiment. Data collection and evaluation has been carried out using the robot welding facility. To measure the bead height as shown in Figure 1, the specimen was cut transversely from the middle position using a wirecutting machine. The selection of the electrode wire should be based principally upon matching the mechanical properties and physical characteristics of the base metal. Secondary consideration should be given to items such as the equipment to be used. The weld size and existing electrode inventory $1.2 \emptyset$ flux-cored wire diameters and $100 \% \mathrm{CO}_{2}$ shielding gas was employed in experiment.

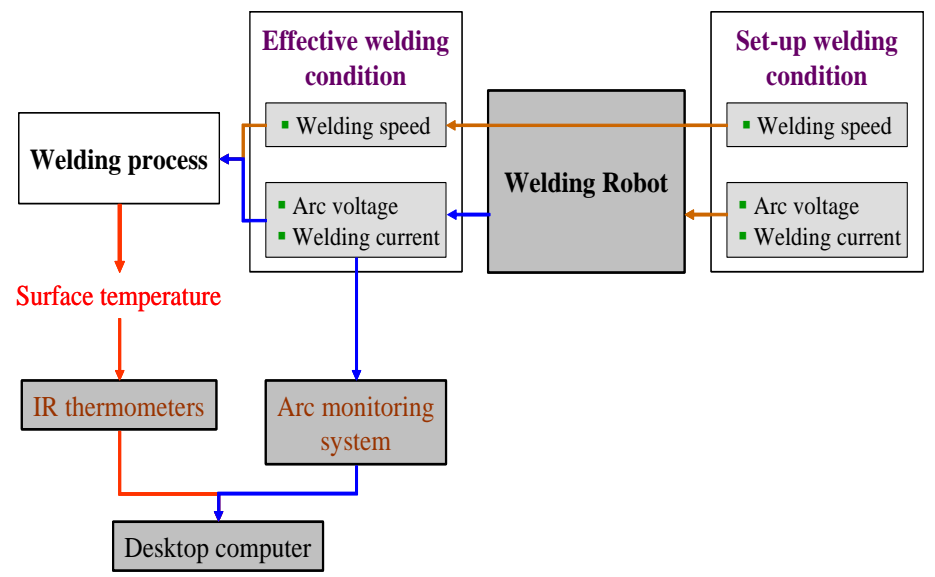

Fig. 1: Block diagram for control system for GMA welding process control system.

\section{Development of on-line empirical model for bead height}

Generally, the development of formalized approach for procedure optimization should be included to establish combination of more welding parameters which would produce good weld quality. In this study, the response function redefined by using additional input parameter about surface temperature and represented as follows:

$$
Y=f\left(S, V, C, T_{1}, T_{2}, T_{3}\right)
$$

\subsection{On-Line Interaction model}

To develop the on-line interaction model, all chosen welding parameters and interaction factors are given below:

$$
\begin{aligned}
Y= & k_{0}+k_{1} S+k_{2} V+k_{3} C+k_{4} T_{1}+k_{5} T_{2}+k_{6} T_{3}+k_{12} S V+k_{12} S C+k_{14} S T_{1}+k_{15} S T_{2}+k_{16} S T_{3}+k_{23} V C \\
& +k_{24} V T_{1}+k_{25} V T_{2}+k_{26} V T_{3}+k_{34} C T_{1}+k_{35} C T_{2}+k_{36} C T_{3}+k_{45} T_{1} T_{2}+k_{46} T_{1} T_{3}+k_{55} T_{2} T_{3}+k_{123} S V C \\
& +k_{124} S V T_{1}+k_{125} S V T_{23}+k_{145} S T_{1}+k_{126} S V T_{3}+k_{134} S C T_{1}+k_{135} S C T_{2}+k_{136} S C T+k_{145} S T_{1} T_{2} \\
& +k_{146} S T_{1} T_{3}+k_{156} S T_{2} T_{3}+k_{234} V C T_{1}+k_{235} V C T_{2}+k_{236} V C T_{3}+k_{345} C T_{1} T_{2}+k_{346} C T_{1} T_{3}+k_{456} T_{1} T_{2} T_{3}
\end{aligned}
$$


The following on-line interaction model for bead height was developed and presented as following:

$$
\begin{aligned}
H_{I}= & 14.647-0.482 S+0.67 V 8-0.012 C_{2}-0.0126 T_{1}+0.002 T-0.008 T 0.001 S C+1.911 \times 10^{-5} S V T_{1} \\
& -3.1 \times 10^{-5} S V T_{2}-6.0 \times 10^{-6} S V T_{3}^{-7}+2.971 \times 10^{-7} S T_{2} T_{3}-8.4 \times 10 V C T_{1}+6.651 \times 10^{-7} V C T_{2} \\
& +7.471 \times 10^{-8} V T_{1} T_{3}-2.0 \times 10^{-7} V T_{2} T_{3}+7.431 \times 10^{-9} C T_{1} T_{3}+2.286 \times 10^{-9} T_{1} T_{2} T_{3}
\end{aligned}
$$

Figure 2 presents comparison between the predicted and measured bead height using on-line interaction model. It is shown that the bead height using on-line interaction model is also good performance. Figure 3 shows the error of predicted results of bead height according to on-line interaction model. Table 1 represents performance of on-line interaction models for predicting bead height. As shown in Table 1, prediction of bead height was shown about 70.93\% in PAM.

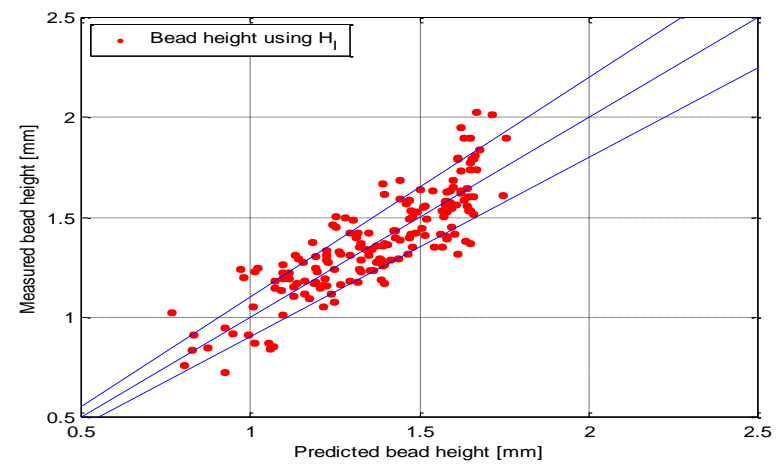

Fig. 2: Comparison between the predicted and measured bead height using on -line interaction model.

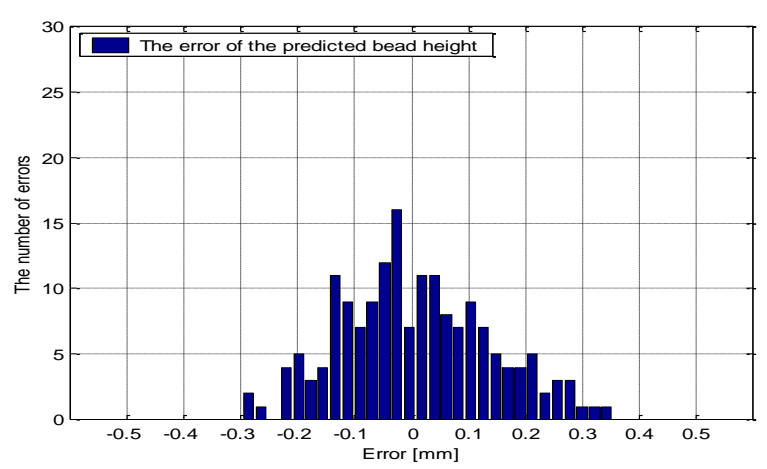

Fig. 3: The error of the predicted bead height using online interaction model.

Table 1: Performance of on-line interaction model.

\begin{tabular}{|c|c|}
\hline Performance & Bead height $\left(H_{I}\right)$ \\
\hline PAM $(\%)$ & 70.9302 \\
\hline Standard deviation & 0.1322 \\
\hline
\end{tabular}

\subsection{On-Line Quadratic Model}

To develop the on-line quadratic model, the response bead height can be shown as bellows:

$$
\begin{aligned}
Y= & k_{0}+k_{1} S+k_{2} V+k_{3} C+k_{4} T_{1}+k_{5} T_{2}+k_{6} T_{3}+k_{12} S V+k_{13} S C+k_{14} S T_{1}+k_{15} S T_{2}+k_{16} S T_{3}+k_{23} V C \\
& +k_{24} V T_{1}+k_{25} V T_{2}+k_{26} V T_{3}+k_{35} C T_{2}+k_{36} C T_{3}+k_{45} T_{1} T_{2}+k_{46} T_{1} T_{3}+k_{56} T_{2} T_{3}+k_{11} S^{2}+k_{22} V^{2} \\
& +k_{33} C^{2}+k_{44} T_{1}^{2}+k_{55} T_{2}^{2}+k_{66} T_{3}^{2}
\end{aligned}
$$

The following on-line quadratic model for bead height was developed and presented:

$$
\begin{aligned}
H_{Q} & =9.341-0.265 S+0.748 \mathrm{~V}+0.044 \mathrm{C}-0.030 T_{1}+0.013 T_{2}-0.006 T_{3}-0.030 \mathrm{SV}+0.002 \mathrm{SC} \\
& +3.70 \times 10^{-4} S T_{1}+2.810 \times 10^{-4} S T_{2}+2.277 \times 10^{-4} S T_{3}-1.9 \times 10^{-5} C T_{1}-3.8 \times 10^{-5} C T_{2}+6.854 \\
& \times 10^{-5} C T_{3}-0.003 S^{2}-0.007 V^{2}-1.562 \times 10^{-4} C^{3}+8.089 \times 10^{-6} T_{1}^{2}-4.3 \times 10^{-7} T_{2}^{2}-3.8 \times 10^{-6} T_{3}^{2}
\end{aligned}
$$

Figure 4 indicates comparison between the predicted and measured bead height using on-line quadratic model. It is shown that the bead height using on-line quadratic model is also good performance. Figure 5 shows the error of predicted results of bead height for on-line quadratic model. Table 2 represents performance of on-line quadratic models for predicting bead height. The values of PAM for bead height model are about $76.74 \%$. It can be concluded that procedure optimization 
for GMA welding process such as non-linear optimization to identify the welding parameter should be required Artificial Intelligence (AI) techniques such as neural network, fuzzy theory and so on.

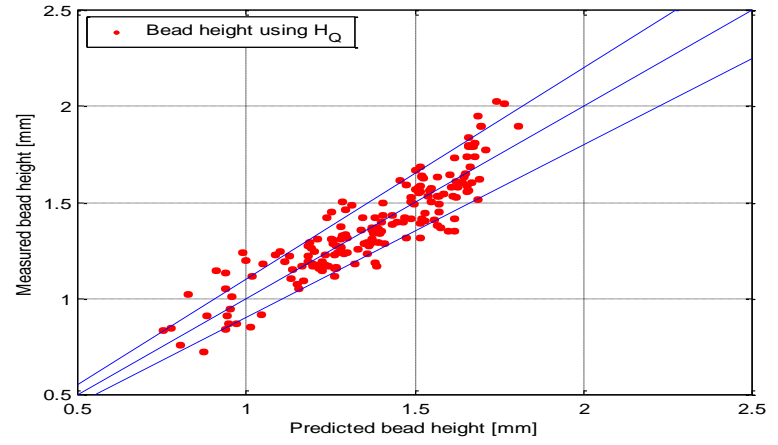

Fig. 4: Comparison between the predicted and measured bead height using on-line quadratic model.

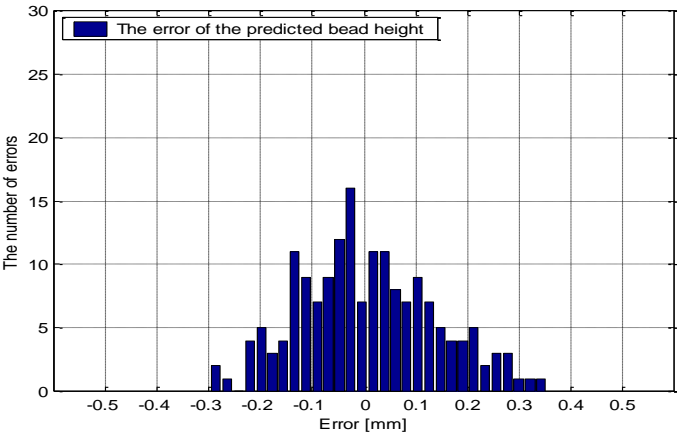

Fig. 5: The error of the predicted bead height using online quadratic model.

Table 2: Performance of on-line quadratic model.

\begin{tabular}{|c|c|}
\hline Performance & $H_{Q}$ \\
\hline PAM $(\%)$ & 76.7442 \\
\hline Standard deviation & 0.1108 \\
\hline
\end{tabular}

\section{Result and Discussion}

To select the most accurate model, additional experiments were carried out. The values of the three welding parameters were chosen for the additional experimental runs. Specially, arc voltage and welding current were changed during automatic GMA welding process to survey the reflation of the developed models according to change the welding condition. The measured position of infrared thermometer was same as the original experiments. Other experiment conditions have been fixed. The welding conditions including CTWD, gas flow rate, welding speed, arc current and welding voltage were employed as the input parameters. The output parameter is the bead height calculated by each developed model and the corresponding errors of prediction. From the calculated results from the developed models which based on additional experiments for this study, it is evident from two developed models that the reasonable agreement between experimental and calculated bead height is shown even when the scatter about the calculated results using two empirical equations (on-line interaction and on-line quadratic models) is considerable. Based on the on-line quadratic model that determines a given bead height and provides useful guidelines for systems which control bead height, a limited range of welding conditions, the effect of each welding parameters and their significant interactions on bead height were computed and plotted. To compare the performance of on-line empirical models, comparisons with the measured results of bead height have been made.

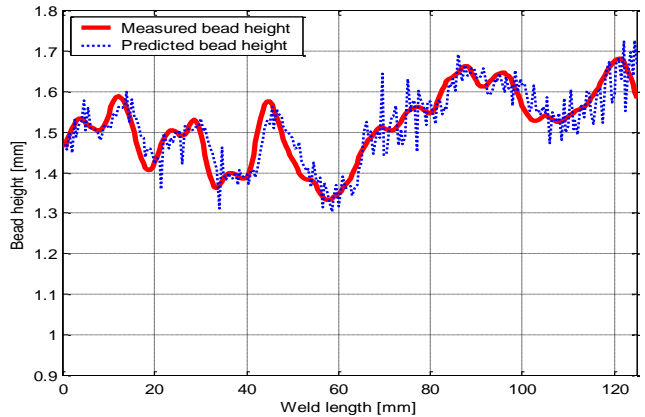

(a) Trial No. 1

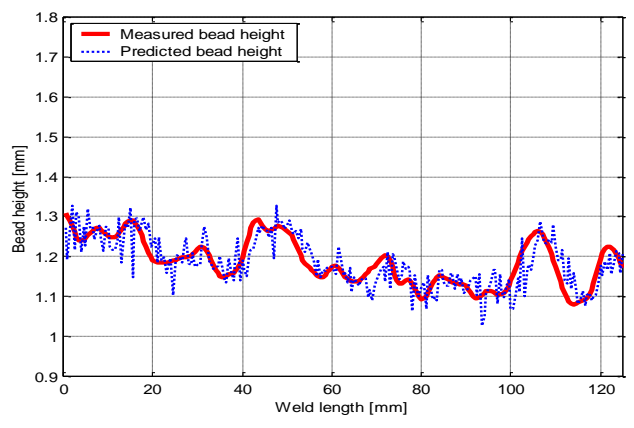

(b) Trial No. 2

Fig. 6: Comparison of the measured and predicted bead height using the developed on-line quadratic model. 
The performance evaluations of developed on-line models have been completed for the bead height in trials No. 1 and No. 2. Figure 6 shows the comparison of the measured and predicted bead height through the developed on-line quadratic model. As shown in Figure 6, the values of predicted bead height using the developed on-line quadratic model is much closed to the values of the measured bead height.

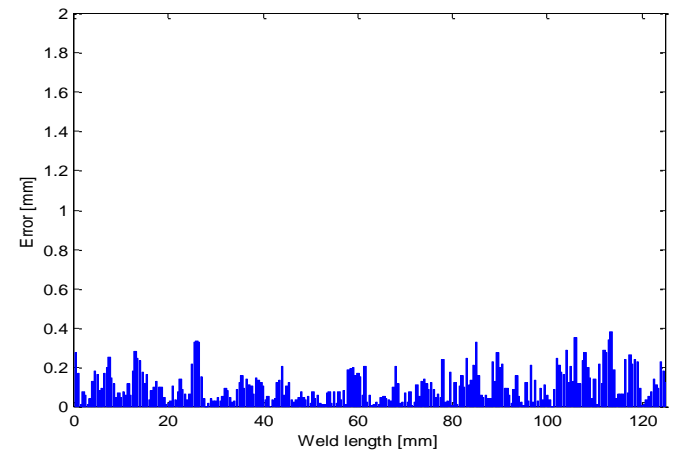

(a) Trial No. 1

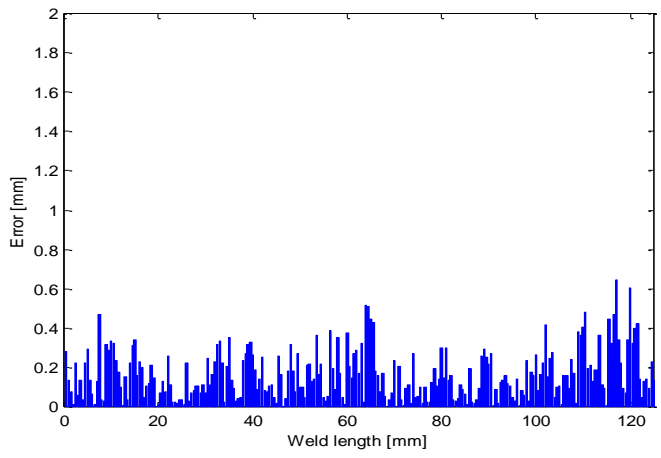

(b) Trial No. 2

Fig. 7: The error of the predicted bead height using the developed on-line quadratic model.

Figure 7 shows the error of predicted bead height using the developed on-line quadratic model. Figures 6-7 present the comparison of the measured and predicted bead height using the on-line quadratic model. In addition, the error of the predicted bead height using the developed on-line quadratic model is concentrated immensely in lower values. Therefore, it is verified that the performance of the developed on-line quadratic model is higher than the developed on-line interaction model.

Table 3: Performance of on-line empirical model for prediction of the bead height.

\begin{tabular}{|c|c|c|c|}
\hline \multicolumn{2}{|c|}{} & Trial No. 1 & Trial No. 2 \\
\hline \multirow{3}{*}{ Bead height } & PAM (\%) & 100 & 98.8 \\
\cline { 2 - 4 } & Standard deviation & 0.0417 & 0.0407 \\
\cline { 2 - 4 } & Average error & 0.0328 & 0.0318 \\
\hline
\end{tabular}

For further verification, the comparison of the measured and predicted bead height used the developed on-line quadratic model has been performed and represented in Table 3 with PAM, standard deviation and average error. The developed model has predicted very accurately. In the comparison of standard deviation and average error, the predicted bead height showed the most concentrated distributions. Eventually, it was concluded that the developed on-line models have a predictive ability that is superior to the other models such as the off-line empirical models.

\section{Conclusions}

The one-line empirical models to predict optimal welding parameters on the required weld height and to investigate the effects of welding parameters on the bead height for the automatic GMA welding process has been developed. Empirical equations (on-line interaction and on-line quadratic model) can find the interrelationship between welding parameters and bead height for the automatic GMA welding process. The comparison with values of coefficient of multiple correlations for the developed models presents no differences, which indicates that all equations are reasonably suitable. The developed two on-line empirical models are able to predict the optimal welding parameters required to achieve desired bead height and weld criteria, help the development of automatic control system and expert system and establish guidelines and criteria for the most effective joint design. A rule-based expert system can be incorporated with the developed neural network system to integrate an optimized system for the automatic GMA welding process. It has been realized that with the use of the developed system, the prediction of bead height becomes much simpler to even a novice user who has no prior knowledge of the automatic GMA welding process and optimization techniques.

\section{Acknowledgements}


This research was financially supported by the Ministry of Education (MOE) and National Research Foundation (NRF) of Korea through the Human Resource Training Project for Regional Innovation. (No. 2013H1B8A2032082).

\section{References}

[1] G. E. Cook, K. Andersen, and R. J. Barrett, "Feedback and adaptive control in welding," in Proceedings of the 2nd International Conference on Trends in Welding Research, 1988, pp. 891-903.

[2] H. B. Smartt, "Arc-welding process," in Welding: Theory and Practice, Elsevier Science Publishers, 1990, pp. $175-208$.

[3] G. Doumanidis, M. Hale, and D. E. Hardt, "Multivariable control of arc welding processes, advances in welding science and technology," in Proceedings of an International Conference on Trends in Welding Research, Gatlinburg, Tennessee, USA, 18-22, May, 1986, pp. 449-457.

[4] J. J. Hunter, G. W. Bryce, and J. Doherty, "On-line control of the arc welding process," in Proceedings of the 2nd International Conference on Computer Technology in Welding, Cambridge, UK, 1988, vol. 37, pp. 1-12.

[5] R. W. Richardson, A. Gutow, R. A. Anderson, and D. F. Farson, "Coaxial weld pool viewing for process monitoring and control," Welding Journal, vol. 63, no. 3, pp. 43-50, 1984. 